\title{
BMJ Open Mortality and potential years of life lost attributable to non-optimal glycaemic control in men and women with diabetes in the United Arab Emirates: a population-based retrospective cohort study
}

Saif Al-Shamsi, ${ }^{\oplus 1}$ Romona Devi Govender, ${ }^{2}$ Elpidoforos S Soteriades ${ }^{3}$

To cite: Al-Shamsi S, Govender RD, Soteriades ES. Mortality and potential years of life lost attributable to nonoptimal glycaemic control in men and women with diabetes in the United Arab Emirates: a population-based retrospective cohort study. BMJ Open 2019;9:e032654. doi:10.1136/ bmjopen-2019-032654

- Prepublication history for this paper is available online To view these files, please visit the journal online (http://dx.doi org/10.1136/bmjopen-2019032654).

Received 28 June 2019 Revised 12 August 2019 Accepted 27 August 2019
Check for updates

(C) Author(s) (or their employer(s)) 2019. Re-use permitted under CC BY-NC. No commercial re-use. See rights and permissions. Published by BMJ.

For numbered affiliations see end of article.

Correspondence to

Dr Saif Al-Shamsi;

salshamsi@uaeu.ac.ae

\section{ABSTRACT}

Objectives Numerous studies reported that achieving near-normal glycated haemoglobin $(\mathrm{HbA1c})$ levels in patients with diabetes may delay or even prevent vascular complications. However, information regarding the impact of non-optimal $\mathrm{HbA} 1 \mathrm{c}$ control on adverse health outcomes in an Arab population is unknown. The aim of this study was to estimate the fraction of deaths and potential years of life lost (PYLL) attributable to non-optimal HbA1c control among Emirati men and women with diabetes in the United Arab Emirates (UAE).

Design A retrospective cohort study.

Setting This study was conducted in outpatient clinics at a tertiary care centre in Al Ain, UAE, between April 2008 and September 2018.

Participants The sample comprised of 583 adult UAE nationals, aged $\geq 18$ years, with diabetes. Overall, $57 \%$ $(n=332)$ of the study participants were men and $43 \%$ $(n=251)$ were women.

Exposure Non-optimal $\mathrm{HbA1c}$ control, defined as $\mathrm{HbA} 1 \mathrm{c} \geq 6.5 \%$.

Primary outcome measure All-cause mortality, defined as death from any cause.

Results At the end of the 9-year follow-up period, 86 (14.8\%) participants died. Overall, up to $33 \%$ (95\% Cl $2 \%$ to $63 \%$ ) of deaths were attributable to non-optimal $\mathrm{HbA1c}$ control among patients with diabetes mellitus (DM). Stratified by sex, the adjusted fraction of avoidable mortality was $17 \%(95 \% \mathrm{Cl}-23 \%$ to $57 \%)$ for men and $50 \%(95 \% \mathrm{Cl} 3 \%$ to $98 \%)$ for women. Both deaths and PYLL attributable to non-optimal $\mathrm{HbA1c}$ control were higher in women compared with men.

Conclusions Up to one-third of all deaths in adult UAE nationals with DM could be attributed to non-optimal $\mathrm{HbA1c}$ control. Effective sex-specific interventions and healthcare quality-improvement programmes should urgently be implemented.

\section{INTRODUCTION}

The WHO estimates that type 2 diabetes mellitus (DM) was the seventh leading cause
Strengths and limitations of this study

- This is the first long-term observational cohort study to estimate the fraction of deaths and potential years of life lost attributable to non-optimal glycated haemoglobin control in adult United Arab Emirates (UAE) men and women with diabetes.

- The diabetes status was determined at baseline and could have changed during the follow-up period, which might have impacted our estimate of the population-attributable fraction (PAF).

- This study was conducted in outpatient clinics of a single tertiary care hospital; therefore, our results may not be applicable to the general population in the UAE.

- Data on other confounding variables that could have impacted study outcomes, such as socioeconomic status, dietary habit and physical activity, were unavailable.

- The Cls for the PAF were wide, indicating that the sample size was relatively small.

of death worldwide in 2016. ${ }^{1}$ The global prevalence of DM among adults has more than doubled over the past four decades and continues to rise. ${ }^{1}$

The United Arab Emirates (UAE) has witnessed substantial economic development since the discovery of oil half a century ago. This increased prosperity has altered the health profile in the UAE with improved access to modern healthcare services and universal health insurance for its citizens. ${ }^{2}$ In the UAE, life expectancy at birth is slowly improving and the most recent estimates for men and women were 76.4 and 80.2 years, respectively, in $2017 .{ }^{3}$ However, this dramatic economic transformation has also led to major lifestyle changes among its residents. Sedentary lifestyles and unhealthy dietary 
habits have resulted in the UAE having one of the highest prevalence of DM worldwide with an age-adjusted prevalence of $17.3 \%$ in $2017 .^{4}$

DM substantially increases the risk of cardiovascular disease (CVD), renal disease, visual impairment and peripheral neuropathy, ${ }^{5-7}$ as well as the risk of premature death. ${ }^{8}$ The WHO estimates that around half of all global deaths in patients with DM aged under 70 years are attributable to poor glycaemic control, ${ }^{1}$ while the proportion of all deaths due to DM among UAE residents aged under 60 years was $69.1 \%{ }^{4}$

There is strong evidence that achieving near-normal glycated haemoglobin (HbA1c) levels in DM significantly reduces morbidity and mortality. ${ }^{9}$ However, despite this evidence, less than half of patients with DM achieve their HbA1c target. A UAE study, in 2015, reported that only $38 \%$ of adult patients with DM had achieved the HbAlc target of $<7 \%$ and that poor glycaemic control was higher in females and patients aged $>60$ years. ${ }^{10}$ This result was similar to a US study, conducted more than a decade ago, which reported that only $34 \%$ of patients with DM had achieved optimal glycaemic control. ${ }^{11}$

The proportion of deaths avoided if patients had achieved the HbA1c target can be quantified by measuring the impact of a specific risk factor on public health, and is described as the population-attributable fraction (PAF) ${ }^{12}$ Since no evidence exists regarding the impact of non-optimal HbA1c control on mortality among Arab patients with diabetes, the aim of this study was to estimate the fraction of deaths and potential years of life lost (PYLL) attributable to non-optimal HbA1c control in UAE nationals with DM.

\section{METHODS}

This was a retrospective cohort study based on data obtained from electronic medical records of UAE nationals with DM, registered at outpatient clinics at Tawam Hospital in Al Ain, UAE, between 01 April 2008 and 31 December 2008. Tawam Hospital is a tertiary care hospital, which provides healthcare services to approximately 19000 adult UAE nationals with DM of Al Ain, the second-largest city in the Emirate of Abu Dhabi, UAE. We included consecutive patients aged $\geq 18$ years who had serum HbAlc level $\geq 6.5 \%$, a diagnosis established by a physician or been receiving medications for DM (eg, sulfonylurea, thiazolidinedione, dipeptidyl peptidase- 4 inhibitor, biguanide or insulin). Patients with incomplete data on serum HbA1c levels and missing history of DM at baseline were excluded (figure 1). Annual follow-up data were obtained between the baseline visits in 2008 and 30 September 2018.

\section{Definitions of clinical variables and outcomes}

Hypertension (HTN) was defined as systolic blood pressure $\geq 140 \mathrm{~mm} \mathrm{Hg}$, diastolic blood pressure $\geq 90 \mathrm{~mm} \mathrm{Hg}$ or the use of antihypertensive medications. ${ }^{13}$ Fasting lipid profiles were measured using

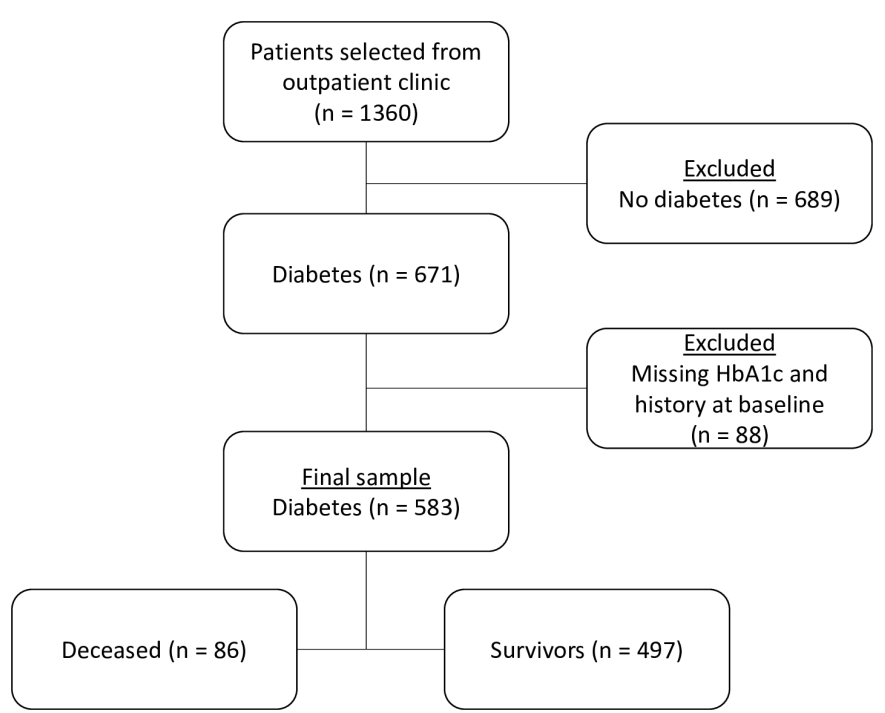

Figure 1 Flow chart of the study population. HbA1c, glycated haemoglobin.

standard methods and the UniCel DxC-800 Synchron Clinical System (Beckman Coulter, Brea, California, USA). Dyslipidaemia was defined as the presence of one or more of the following: triglyceride $\geq 1.69 \mathrm{mmol} / \mathrm{L}$, total cholesterol $\geq 5.17 \mathrm{mmol} / \mathrm{L}$, low-density lipoprotein cholesterol $\geq 3.36 \mathrm{mmol} / \mathrm{L}$, high-density lipoprotein cholesterol $<1.03 \mathrm{mmol} / \mathrm{L}$ or documented treatment with lipid-lowering medications. ${ }^{14}$ Body mass index (BMI) was calculated as weight $(\mathrm{kg})$ divided by height $(\mathrm{m})$ squared and obesity was defined as $\mathrm{BMI} \geq 30 \mathrm{~kg} / \mathrm{m}^{2}$. Smoking history was positive if there was a current or history of smoking of tobacco. Patients were considered as having CVD if they had a diagnosis of coronary heart disease (angina, prior myocardial infarction, angioplasty of the coronary arteries or coronary artery surgery), cerebrovascular accidents or peripheral vascular disease. History of cancer was defined as an established diagnosis of cancer of any type. Chronic kidney disease (CKD) was defined as an estimated glomerular filtration rate $<60 \mathrm{~mL} /$ $\min / 1.73 \mathrm{~m}^{2}{ }^{15} \mathrm{HbA} 1 \mathrm{c}$ was assessed by the medical laboratory department at Tawam Hospital using an automated analyser (Integra 400 Plus; Roche Diagnostics GmbH, Mannheim, Germany). Non-optimal HbAlc control was defined as $\mathrm{HbA} 1 \mathrm{c} \geq 6.5 \%{ }^{16}$

Mortality data were collected retrospectively during the follow-up period until 30 September 2018. Death was defined as death from any cause and was confirmed through the review of medical records and death certificates. Time to event was calculated for each patient as the difference between the date of the inclusion into the study and the date of death, or the date of the last outpatient clinic visit, whichever occurred first.

\section{Statistical analysis}

Data were expressed as mean $\pm \mathrm{SD}$ or percentages. The baseline characteristics were compared by sex using the 
independent samples t-test for continuous variables and Fisher's exact test (two-tailed) for categorical variables.

For this analysis, we estimated Cox proportional hazards regression models adjusted for age (continuous), sex, HTN, dyslipidaemia, cancer, smoking, CVD, CKD, obesity and DM medications to estimate HRs and $95 \%$ CI for the association between non-optimal HbAlc control and mortality. The assumption of proportionality was met on the basis of log-log plots.

The main exposure of interest was non-optimal HbAlc control. The outcomes of interest were death and PYLL. PYLL was calculated by subtracting the average age of death from the average life expectancy (for UAE nationals in $2017^{3}$ ), and then by multiplying this number by the number of non-optimal HbA1c control-attributable deaths.

We estimated the PAF associated with non-optimal HbAlc control using the attributable fraction package for censored survival data in R software. ${ }^{17}$ The PAF for HbAlc $\geq 6.5 \%$ was adjusted for age (continuous), sex, HTN, dyslipidaemia, cancer, smoking, CVD, CKD, obesity and DM medications. The PAF was then used to calculate deaths and PYLL that could be averted if HbA1c control was optimal. The proportion of mortality cases and PYLL that could have been prevented if all patients had optimal HbAlc control was indicated by a positive PAF. Conversely, a negative PAF is the proportion of mortality cases and PYLL that could have additionally occurred if patients had optimal HbA1c.

All statistical analyses were performed using R software V.3.5.2 (The R Foundation, Vienna, Austria) and IBM SPSS software V.25. All p values were two-tailed, and $<0.05$ were considered statistically significant.

\section{Patient and public involvement}

There was no patient or public involvement in the design and conduct of the study.

\section{RESULTS}

\section{Baseline characteristics}

The analysis in the current study included 583 subjects with DM (figure 1), with a median follow-up of 9.1 years (IQR 8.1-9.7 years). Approximately 57\% of the study participants were men (table 1 ). The mean age of participants at baseline was 58.4 years (SD 12.4 years) and the mean \pm SD HbAlc was $7.85 \% \pm 1.93 \%$. Approximately $84 \%$ $(487 / 583)$ of patients were on medications for DM. Of these treated patients, only 23\% (113/487) had optimal HbAlc control. The baseline characteristics stratified by sex are presented in table 1 . Men were older at baseline; more frequently had a history of smoking, dyslipidaemia and CVD; but less frequently had a history of obesity than women. HbA1c at baseline was significantly higher in

\begin{tabular}{|c|c|c|c|c|}
\hline Characteristic & Total $(n=583)$ & Women $(n=251)$ & Men $(n=332)$ & P value* \\
\hline Age (years), mean \pm SD & $58.4 \pm 12.4$ & $57.0 \pm 11.4$ & $59.4 \pm 13.0$ & 0.021 \\
\hline \multicolumn{5}{|l|}{ Age (years), n (\%) } \\
\hline$\leq 39$ & $50(8.6)$ & $18(7.2)$ & $32(9.6)$ & $<0.001$ \\
\hline $40-54$ & $155(26.6)$ & $88(35.1)$ & $67(20.2)$ & \\
\hline $55-64$ & $179(30.7)$ & $77(30.7)$ & $102(30.7)$ & \\
\hline$\geq 65$ & $199(34.1)$ & $68(27.1)$ & $131(39.5)$ & \\
\hline Women, n (\%) & $251(43.1)$ & & & \\
\hline DM medications, n (\%) & $487(83.5)$ & $202(80.5)$ & $285(85.8)$ & 0.091 \\
\hline $\mathrm{HbA1c}(\%)$, mean $\pm \mathrm{SD}$ & $7.85 \pm 1.93$ & $7.53 \pm 1.75$ & $8.09 \pm 2.02$ & $<0.001$ \\
\hline 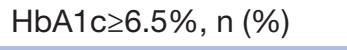 & $470(80.6)$ & $195(77.7)$ & $275(82.8)$ & 0.138 \\
\hline \multicolumn{5}{|l|}{ Comorbidities, n (\%) } \\
\hline Smoking history & $108(18.5)$ & $2(0.8)$ & $106(31.9)$ & $<0.001$ \\
\hline Obesity† & $276(47.3)$ & $151(60.4)$ & $125(37.7)$ & $<0.001$ \\
\hline HTN & $495(84.9)$ & $209(83.3)$ & $286(86.1)$ & 0.352 \\
\hline Dyslipidaemia & $534(91.6)$ & $219(87.3)$ & $315(94.9)$ & 0.001 \\
\hline CVD & $150(25.7)$ & $33(13.1)$ & 117 (35.2) & $<0.001$ \\
\hline Cancer & $46(7.9)$ & $21(8.4)$ & $25(7.5)$ & 0.757 \\
\hline CKD & $89(15.3)$ & $31(12.4)$ & $58(17.5)$ & 0.103 \\
\hline
\end{tabular}

*The independent samples t-test was used to calculate $p$ values for continuous variables, and Fisher's exact test (2-tailed) was used to calculate $p$ values for categorical variables.

$\dagger \mathrm{n}=582$.

CKD, chronic kidney disease; CVD, cardiovascular disease; DM, diabetes mellitus; HbA1c, glycated haemoglobin; HTN, hypertension. 
Table 2 HRs and population-attributable fraction of allcause mortality associated with $\mathrm{HbA} 1 \mathrm{c} \geq 6.5 \%$

\begin{tabular}{lll}
\hline & All-cause mortality & P value \\
\hline Events/non-events & $86 / 497$ & \\
HR $^{*}(95 \% \mathrm{Cl})$ & $1.37(0.76$ to 2.47$)$ & 0.300 \\
HR† $(95 \% \mathrm{Cl})$ & $1.24(0.69$ to 2.25$)$ & 0.471 \\
HR $\neq(95 \% \mathrm{Cl})$ & $1.79(0.95$ to 3.39$)$ & 0.071 \\
\hline PAF $\neq, \%(95 \% \mathrm{Cl})$ & $33(2$ to 63$)$ & 0.039 \\
\hline
\end{tabular}

*Univariable.

†Adjusted for age (continuous) and sex.

łAdjusted for age (continuous), sex, HTN, dyslipidaemia, cancer, smoking, CVD, CKD, obesity and DM medications.

CKD, chronic kidney disease; CVD, cardiovascular disease; DM, diabetes mellitus; HbA1c, glycated haemoglobin; HTN, hypertension; PAF, population-attributable fraction.

men than in women $(\mathrm{p}<0.001)$. There were no significant differences by sex in non-optimal HbAlc control at baseline $(\mathrm{p}=0.138)$.

\section{Mortality and PYLL}

At the end of the follow-up period, 86 (14.8\%) deaths occurred. The multivariable-adjusted HR for all-cause mortality comparing patients with and without optimal HbA1c control was 1.79 (95\% CI 0.95 to 3.39). The multivariable-adjusted PAF for deaths due to non-optimal HbA1c control was $33 \%$ (95\% CI 2\% to 63\%) (table 2). Overall, 28 (95\% CI 2 to 54) deaths could have been prevented if the HbA1c of all participants in the study had been maintained in the optimal range. Similarly, 190 (95\% CI 12 to 363) PYLL could have been prevented if the HbAlc was optimal in all patients.

When stratified by sex, the confounder-adjusted fraction of avoidable mortality was $17 \%(95 \%$ CI $-23 \%$ to $57 \%$ ) for men and $50 \%$ (95\% CI 3\% to 98\%) for women. Shown in figures 2 and 3, respectively, are the deaths and PYLL attributable to non-optimal HbA1c control, by age category and sex. The avertable deaths were higher for the

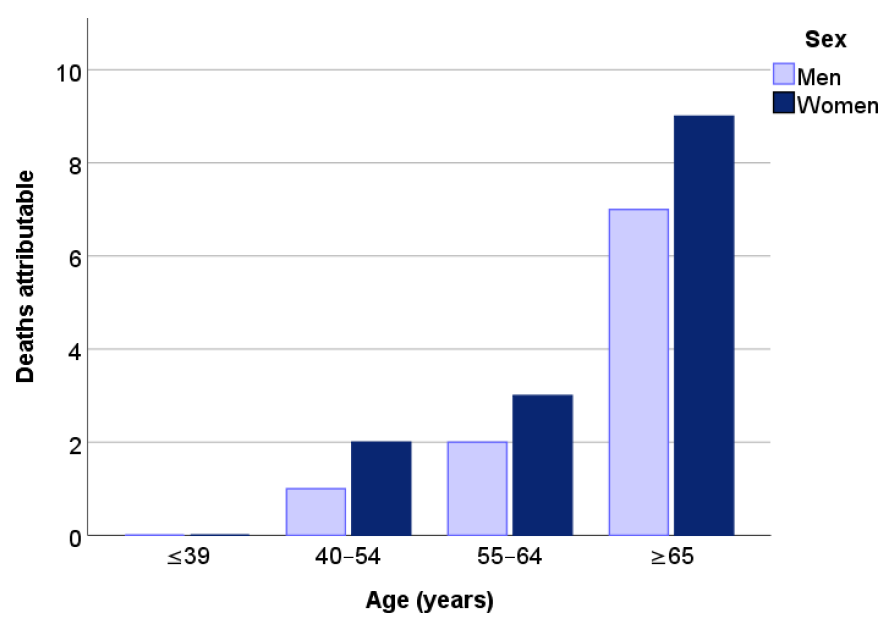

Figure 2 Deaths attributable to non-optimal $\mathrm{HbA} 1 \mathrm{c}$ by age and sex. HbA1c, glycated haemoglobin.

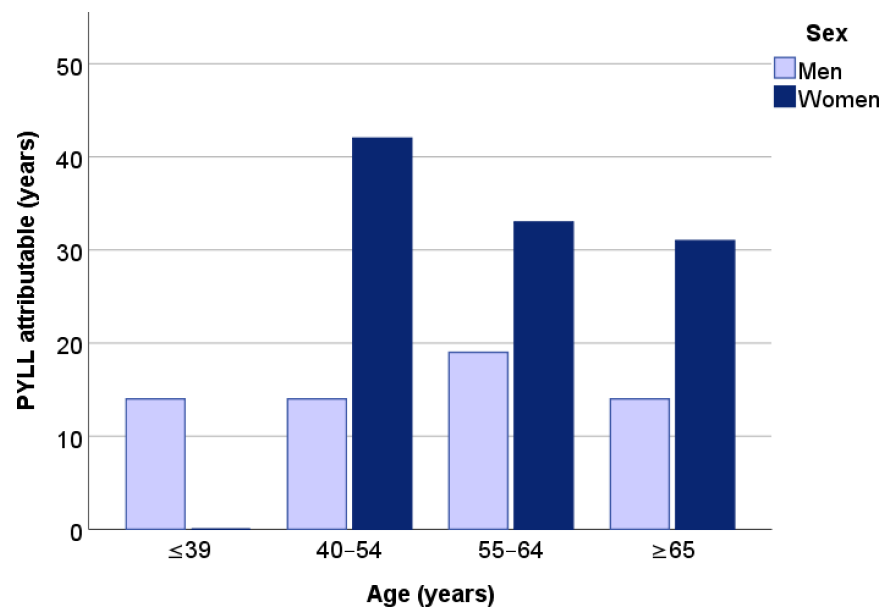

Figure 3 PYLL attributable to non-optimal $\mathrm{HbA1c}$ by age and sex. HbA1c, glycated haemoglobin; PYLL, potential years of life lost.

older age groups in both men and women. In women, the avertable PYLL was higher in the 40-54 years age group; however, in men, the PYLL was higher in the 55-64 years age group. Overall, both deaths and PYLL attributable to non-optimal HbAlc control were higher in women than in men.

Extrapolating from our cohort to the approximately 155000 adult UAE nationals with $\mathrm{DM}^{4}$ and assuming that this population is comparable to our study population, based on our results, we estimated that approximately 22900 deaths would have been observed within 9 years among the Emirati population with DM. Of these total deaths, around 7600 deaths would be attributable to non-optimal HbAlc control.

\section{DISCUSSION}

This long-term observational cohort study suggests that among adult Emiratis with DM, about one-third of all deaths over the 9-year period could have been prevented with optimal HbA1c control. By extrapolating our results to the UAE national population with DM, we estimated the burden of non-optimal HbAlc control among Emiratis. In the approximately 155000 adult UAE nationals with $\mathrm{DM},{ }^{4}$ this would correspond to about 840 deaths that could have potentially been avoided annually.

There is only one comparable longitudinal study in the international literature that estimated the attributable risk associated with HbA1c levels for all-cause mortality in patients with DM. The cardiometabolic Valencian (ESCARVAL-RISK) study conducted on a large general-practice cohort of Spanish patients with DM reported the PAF associated with uncontrolled diabetes as $13.6 \%$ (95\% CI $4.0 \%$ to $23.9 \%)$ for all-cause mortality. ${ }^{18}$ This estimate was much lower than what was observed in our study. This difference may be explained by different population characteristics and follow-up time between the two studies (patients with CVD were excluded from the ESCARVAL-RISK study and had a follow-up of 3.3 
years vs $26 \%$ with a history of CVD and a follow-up of 9.1 years in our study).

To the best of our knowledge, the PAF linked to non-optimal HbAlc control by sex has not been previously reported. We found that more deaths could have been avoided in women with non-optimal HbAlc control than in men. Moreover, in our study, the PAF associated with uncontrolled diabetes was not statistically significantly contributing to the risk of mortality in men. Most previous studies have supported the claim that women with DM are at higher risk for morbidity and mortality compared with men with DM. ${ }^{19-22}$ A meta-analyses of patients with DM reported that women were $50 \%$ more likely to die of fatal coronary heart disease than men. ${ }^{23}$ It is not known why women with DM are at increased risk for morbidity and mortality compared with their male counterparts. One explanation is that CVD risk factors affect women more than men. The Strong Heart Study showed that between women with and without DM, the differences in levels of several CVD risk factors were greater than differences found in men with and without DM. ${ }^{24}$ Another possible explanation is that women with diabetes are less likely than men to have CVD risk factors aggressively treated. ${ }^{25}$ In addition, results from a Korean study on patients with DM showed that compared with men, women were less likely to achieve optimal HbAlc control after 1 year of treatment. ${ }^{26}$ Our findings demonstrate possible sex differences in mortality among Emirati patients with poorly controlled DM and support the urgent need for developing sex-specific tertiary prevention strategies in the UAE.

In our study, patients with DM had a moderate glycaemic control with a mean \pm SD HbAlc of $7.85 \% \pm 1.93 \%$ at baseline and only approximately $19 \%$ of them had optimal HbAlc target of $<6.5 \%$. These results are consistent with other studies carried out in neighbouring Middle Eastern and Western countries, ${ }^{27-30}$ indicating that poor glycaemic control in patients with DM is a serious issue worldwide. A study conducted among 651 patients with DM in Saudi Arabia showed that close to $21 \%$ of patients had optimal HbA1c control. ${ }^{27}$ A nationwide French survey on 4930 patients with DM reported that the mean HbAlc was $7.6 \% \pm 1.6 \%$ and in $27 \%$ of patients, the HbAlc level was $\leq 6.5 \% .{ }^{29}$ Possible reasons for this failure may be multifactorial, such as poor compliance with medications, and therapeutic inertia on the part of healthcare providers. A recently published systematic review reported that the delay in the intensification of therapy following an HbA1c measurement above the target level was longer than 1 year. ${ }^{31}$ Furthermore, the increasing prevalence of multimorbidity among patients often complicates the management of $\mathrm{DM},{ }^{32}$ and may also contribute to suboptimal diabetes management. It is well-known and widely accepted that achieving optimal HbA1c target levels in patients with DM, as outlined in current clinical guidelines, ${ }^{16} 3334$ decreases the risk of vascular complications and improves the life expectancy. ${ }^{6}$ Therefore, future studies, to investigate effective and efficient solutions in improving glycaemic control in patients with DM, should be high on the list of priorities for all decision-makers involved in DM care.

\section{Strengths and limitations}

To the best of our knowledge, this is the first long-term observational cohort study to estimate the fraction of deaths and PYLL attributable to non-optimal HbA1c control in UAE national men and women with DM. The PAF assesses potential health benefits through risk factor control and disease prevention. It is a helpful instrument for effective health communication that may increase both public and healthcare professionals' awareness of DM. It may also aid in decreasing the risk of mortality with the implementation of healthcare quality-improvement programmes by key stakeholders.

This study has several limitations. First, the DM status was determined at baseline and could have changed during the follow-up period, which might have impacted our estimate of the PAF. However, transitions from diabetes to non-diabetes status are rare. ${ }^{35}$ Second, this study was conducted in outpatient clinics of a single tertiary care hospital; therefore, our results may not be applicable to the general population in the UAE. Third, the changes in HbA1c levels and DM treatment over time were not assessed and could have affected the study results. In addition, data on other confounding variables that could have impacted the study outcomes, such as the socioeconomic status, dietary habits and physical activity, were unavailable. Finally, the CIs for the PAF were wide, indicating that the sample size was relatively small; however, our study in an Arab population with a long follow-up duration gives us a better picture of the high burden of diabetes in the Middle East, where data on associated poor glycaemic control is sparse. Additional large multicenter studies are needed to confirm our results.

\section{CONCLUSION}

In conclusion, non-optimal HbA1c control contributed to one-third of all deaths in patients with DM in the UAE. This places a significant burden on the health of Emiratis with DM, their families and the health systems. Effective interventions, such as the training of DM care teams on sex-specific prevention strategies, periodic monitoring of DM and improving patients' education, are essential to increase adherence to management guidelines and should be implemented without delay.

\section{Author affiliations}

${ }^{1}$ Internal Medicine, United Arab Emirates University College of Medicine and Health Sciences, Al Ain, United Arab Emirates

${ }^{2}$ Family Medicine, United Arab Emirates University College of Medicine and Health Sciences, Al Ain, United Arab Emirates

${ }^{3}$ Institute of Public Health, United Arab Emirates University College of Medicine and Health Sciences, Al Ain, United Arab Emirates

Contributors SAS, RDG and ESS contributed to the research idea and design of the study. SAS and RDG contributed to acquisition of data. SAS, RDG and ESS contributed to analysis and interpretation of data, and SAS was responsible for the 
statistical analysis. SAS drafted the manuscript. SAS, RDG and ESS contributed to the critical revision of the manuscript for important intellectual content. All the authors have read and approved the final version of the manuscript.

Funding This work was supported by the College of Medicine and Health Sciences, UAE University (grant number 31M325).

Disclaimer The funders had no role in the study design, data collection and analysis, decision to publish or preparation of the manuscript.

Competing interests None declared.

Patient consent for publication Not required.

Ethics approval Ethical approval for this study was obtained from Tawam Hospital and the UAE University's Research and Ethics Board (CRD 239/13).

Provenance and peer review Not commissioned; externally peer reviewed.

Data availability statement Data are available in a public, open-access repository. Supplementary data and analysis can be accessed from the Mendeley repository(http://dx.doi.org/10.17632/mj94yydtmn.1). All data have been provided in the study and anyone is permitted to use the data provided that the article is properly cited.

Open access This is an open access article distributed in accordance with the Creative Commons Attribution Non Commercial (CC BY-NC 4.0) license, which permits others to distribute, remix, adapt, build upon this work non-commercially, and license their derivative works on different terms, provided the original work is properly cited, appropriate credit is given, any changes made indicated, and the use is non-commercial. See: http://creativecommons.org/licenses/by-nc/4.0/.

\section{REFERENCES}

1. Roglic G. WHO global report on diabetes: a summary. Int $J$ Noncommun Dis 2016;1:3-8.

2. Koornneef E, Robben P, Blair I. Progress and outcomes of health systems reform in the United Arab Emirates: a systematic review. BMC Health Serv Res 2017;17:672.

3. Statistics Centre - Abu Dhabi. Statistical Yearbook of abu dhabi, 2018. Available: https://www.scad.gov.abudhabi/Release\% 20Documents/SYB_2018_EN_9Sep\%20_Chart\%20Correction.pdf [Accessed 18 Mar 2019].

4. International Diabetes Federation. IDF diabetes atlas. 8th eds. Brussels, Belgium: International Diabetes Federation, 2017.

5. Bourne RRA, Stevens GA, White RA, et al. Causes of vision loss worldwide, 1990-2010: a systematic analysis. Lancet Glob Health 2013;1:e339-49.

6. Sarwar N, Gao P, Seshasai SRK, et al. Diabetes mellitus, fasting blood glucose concentration, and risk of vascular disease: a collaborative meta-analysis of 102 prospective studies. Lancet 2010;375:2215-22.

7. Saran R, Li Y, Robinson B, et al. US renal data system 2014 annual data report: epidemiology of kidney disease in the United States. American Journal of Kidney Diseases 2015;66:A7-305. 1.

8. Saydah SH, Eberhardt MS, Loria CM, et al. Age and the burden of death attributable to diabetes in the United States. Am J Epidemiol 2002;156:714-9.

9. Rawshani A, Rawshani A, Franzén S, et al. Risk factors, mortality, and cardiovascular outcomes in patients with type 2 diabetes. $N$ Engl $J$ Med 2018;379:633-44.

10. Ali Malik A. Clinical diabetes care of patients with type 2 diabetes at a major tertiary care hospital in the United Arab Emirates. J Diabetes Metab Disord Control 2015;2.

11. Grant RW, Buse JB, Meigs JB, et al. Quality of diabetes care in U.S. academic medical centers: low rates of medical regimen change. Diabetes Care 2005;28:337-442.

12. Spiegelman D, Hertzmark E, Wand HC. Point and interval estimates of partial population attributable risks in cohort studies: examples and software. Cancer Causes Control 2007;18:571-9.

13. National High Blood Pressure Education Program. The seventh report of the joint National Committee on prevention, detection, evaluation, and treatment of high blood pressure. Bethesda (MD): National heart, lung, and blood Institute (US), 2004. Available: http:// www.ncbi.nlm.nih.gov/books/NBK9630/ [Accessed 18 Mar 2019]

14. Expert Panel on Detection, Evaluation, and Treatment of High Blood Cholesterol in Adults. Executive summary of the third report of the
National cholesterol education program (NCEP) expert panel on detection, evaluation, and treatment of high blood cholesterol in adults (adult treatment panel III). JAMA 2001;285:2486-97.

15. Levin A, Stevens PE, Bilous RW, et al. Kidney disease: improving global outcomes (KDIGO) CKD work group. KDIGO 2012 clinical practice guideline for the evaluation and management of chronic kidney disease. Kidney Int Supp/ 2013:3:1-150.

16. American Diabetes Association. 6. Glycemic Targets: Standards of Medical Care in Diabetes-2019. Diabetes Care 2019;42(Suppl 1):S61-S70.

17. Dahlqwist E, Zetterqvist J, Pawitan $\mathrm{Y}$, et al. Model-Based estimation of the attributable fraction for cross-sectional, case-control and cohort studies using the R package AF. Eur J Epidemiol 2016;31:575-82.

18. Navarro-Pérez J, Orozco-Beltran D, Gil-Guillen V, et al. Mortality and cardiovascular disease burden of uncontrolled diabetes in a registrybased cohort: the ESCARVAL-risk study. BMC Cardiovasc Disord 2018;18:180.

19. Barnett KN, Ogston SA, McMurdo MET, et al. A 12-year follow-up study of all-cause and cardiovascular mortality among 10,532 people newly diagnosed with type 2 diabetes in Tayside, Scotland. Diabet Med 2010;27:1124-9.

20. Natarajan S, Liao Y, Cao G, et al. Sex differences in risk for coronary heart disease mortality associated with diabetes and established coronary heart disease. Arch Intern Med 2003;163:1735-40.

21. Juutilainen A, Kortelainen S, Lehto $S$, et al. Gender difference in the impact of type 2 diabetes on coronary heart disease risk. Diabetes Care 2004:27:2898-904.

22. Roche MM, Wang PP. Sex differences in all-cause and cardiovascular mortality, hospitalization for individuals with and without diabetes, and patients with diabetes diagnosed early and late. Diabetes Care 2013;36:2582-90.

23. Huxley R, Barzi F, Woodward M. Excess risk of fatal coronary heart disease associated with diabetes in men and women: meta-analysis of 37 prospective cohort studies. BMJ 2006;332:73-8.

24. Howard BV, Cowan LD, Go O, et al. Adverse effects of diabetes on multiple cardiovascular disease risk factors in women. The strong heart study. Diabetes Care 1998:21:1258-65.

25. Gouni-Berthold I, Berthold HK, Mantzoros CS, et al. Sex disparities in the treatment and control of cardiovascular risk factors in type 2 diabetes. Diabetes Care 2008:31:1389-91.

26. Choe S-A, Kim JY, Ro YS, et al. Women are less likely than men to achieve optimal glycemic control after 1 year of treatment: a multi-level analysis of a Korean primary care cohort. PLoS One 2018;13:e0196719.

27. Al-Hussein FA. Diabetes control in a primary care setting: a retrospective study of 651 patients. Ann Saudi Med 2008;28:267-71.

28. Rätsep A, Kalda R, Lember M. Meeting targets in type 2 diabetes care contributing to good glycaemic control. A cross-sectional study from a primary care setting in Estonia. Eur $\mathrm{J}$ Gen Pract 2010;16:85-91.

29. Charpentier G, Genès N, Vaur L, et al. Control of diabetes and cardiovascular risk factors in patients with type 2 diabetes: a nationwide French survey. Diabetes Metab 2003;29:152-8.

30. Alvarez Guisasola F, Mavros P, Nocea G, et al. Glycaemic control among patients with type 2 diabetes mellitus in seven European countries: findings from the real-life effectiveness and care patterns of diabetes management (RECAP-DM) study. Diabetes Obes Metab 2008:10 Suppl 1:8-15.

31. Khunti K, Gomes MB, Pocock S, et al. Therapeutic inertia in the treatment of hyperglycaemia in patients with type 2 diabetes: a systematic review. Diabetes Obes Metab 2018;20:427-37.

32. Magnan EM, Palta M, Mahoney JE, et al. The relationship of individual comorbid chronic conditions to diabetes care quality. $B M J$ Open Diabetes Res Care 2015;3:e00080.

33. International Diabetes Federation Guideline Development Group. Global guideline for type 2 diabetes. Diabetes Res Clin Pract 2014;104:1-52.

34. Inzucchi SE, Bergenstal RM, Buse JB, et al. Management of hyperglycemia in type 2 diabetes, 2015: a patient-centered approach: update to a position statement of the American diabetes association and the European association for the study of diabetes. Diabetes Care 2015;38:140-9.

35. Karter AJ, Nundy S, Parker MM, et al. Incidence of remission in adults with type 2 diabetes: the diabetes \& aging study. Diabetes Care 2014;37:3188-95. 